\title{
Comment
}

\section{Operational research and new technology}

Organizations around the world are struggling to come to grips with four major issues: intensifying global competition, a changing workforce, changing rules of competition, and new technology. The one of these that appears to be having the greatest impact on OR is new technology.

OR has a history of coping with and adjusting to technological change. We have become used to spending a great deal of effort to "shoehorn" our work into the limited technology of the day. Much of our advancement owes as much to technological improvement as it does to conceptual leaps from within OR.

What was true in the past, however, does not reflect the present. The powerful networked workstation of the 1990s is to OR like a blank sheet of paper to an artist; it provides us with space for creativity and new directions. Technological constraints have become technological opportunities that are already being exploited as evidenced by (for example) the surge of interest in user interfaces and computer-generated graphics, and group DSS.

Technological change is, however, rarely painless. We, like every other organization, must face some tough issues. The toughest is to recognise that advancing technology has rendered obsolete some concepts we once cherished: it is essential for our future that we do recognise this, and leave our baggage behind.

I would like to see us begin a dialogue on what we should discard. To start things off, I suggest that we no longer automatically reward work that improves algorithmic efficiency. Technology has confused the notion of algorithmic efficiency; there are obvious measurement problems resulting from the variety of hardware/systems available. More importantly, however, in the eyes of most decision-makers, saving CPU time is not very valuable any more - How can we continue to pay a great deal of attention to an objective that most of the world (including our customers) thinks is trivial? Finally, since most data entry is now performed interactively, the traditional measures of algorithmic efficiency which emphasise minimum computation time are no longer very meaningful; since the user cannot enter the data in zero time, a fast algorithm which cannot start until the entire problem has been entered may well be less "efficient" than a slower procedure that can compute as the user enters the problem.

Another consequence of technological change that is particularly challenging and requires more of our attention is the increasing computer-literacy of senior management. This trend will continue - computers are more and more in evidence at all levels of our education system. Further, the rapid expansion of management education programmes has resulted in a large number of senior managers who not only know what OR is, but understand what we do, and may even be capable of doing some serious modelling themselves.

OR has largely ignored the computer/OR-literate manager [although I have heard concerns that some of our best work is no longer identified with OR (e.g. PERT/CPM), and others are concerned that the decisionmaker with the spreadsheet may render much of what we do obsolete]. Our end-users will continue to become increasingly computer-literate; the issue for $O R$ is how we react to this.

Two directions that our response should take are to encourage the modeller/decision-maker, and to exploit the emerging literacy among our customers. One idea I like to encourage and support the decision-maker/ modeller is "end-user OR" - an obvious parallel to enduser computing. Is there a role for "OR centres", where decision-makers can go to obtain advice on their modelling efforts from user-friendly operational researchers? Are there "OR hotlines" in our future? How else can OR encourage and provide assistance and quality control on decision-maker modelling?

Exploiting the computer-literate decision-maker leads us towards symbiotic decision-maker/computerinteractive problem-solving systems. While there is some overlap here with DSS, there is an opportunity to think in broader terms. Executives make more than one decision, and spend much of their time on tasks not involving decision-making. We should think less about individual decisions and more about the contents of the executive's workstation. What about a job support system: a suite of several DSSs plus executive information system, spreadsheet, word processor, data book program, etc., all working with a standard interface?

OR provides a very good training for general management - we should not be surprised when our best practitioners are promoted and become our customers. We should also not be surprised if these new decision-makers are no longer very interested in what we do. An important part of the technological challenge we now face is to learn how to adapt what we do in order to provide more value to technologically-sophisticated users.

PETER BELL

\section{Acknowledgement}

This item was previously circulated as a Letter from the President with IFORS Bulletin No. 7, 3rd quarter 1990. 